\title{
Comparison of Markov Chain Abstraction and Monte Carlo Simulation for the Safety Assessment of Autonomous Cars
}

\author{
Matthias Althoff and Alexander Mergel
}

\begin{abstract}
The probabilistic prediction of road traffic scenarios is addressed. One result is a probabilistic occupancy of traffic participants, the other result is the collision risk for autonomous vehicles when executing a planned maneuver. The probabilistic occupancy of surrounding traffic participants helps to plan the maneuver of an autonomous vehicle, while the computed collision risk helps to decide if a planned maneuver should be executed. Two methods for the probabilistic prediction are presented and compared: Markov chain abstraction and Monte Carlo simulation. The performance of both methods is evaluated with respect to the prediction of the probabilistic occupancy and the collision risk. For each comparison test we use the same models generating the probabilistic behavior of traffic participants, where the generation of these data is not compared to real world data. However, the results show independently of the behavior generation that Markov chains are preferred for the probabilistic occupancy, while Monte Carlo simulation is clearly preferred for determining the collision risk.
\end{abstract}

Index Terms-Safety assessment, threat level, autonomous cars, Markov chains, Monte Carlo simulation, probabilistic occupancy, crash probability, behavior prediction.

\section{INTRODUCTION}

$\mathbf{O}$ $\mathrm{NE}$ of the main objectives of research on autonomous vehicles is to realize the vision of accident-free driving by exclusion of human errors. This can be done by fully autonomous vehicles or partly autonomous vehicles, which only take over the control when an accident is, or is almost inevitable.

In order to assess the safety of a planned maneuver, the prediction of traffic participants is vital for the identification of future threats. In contrast to predictive approaches, nonpredictive methods are based on the recording and evaluation of traffic situations that have resulted in dangerous situations; see e.g. [1], [31]. However, this approach is only suitable for driver warnings. Planned trajectories of autonomous cars cannot be evaluated with this method since the consequences when following these trajectories have to be predicted.

Behavior prediction has been mainly limited to human drivers within the ego vehicle (i.e. the vehicle for which the safety assessment is performed). This is motivated by research on driver assistant systems which warn drivers when dangerous

Matthias Althoff is with the Department of Electrical and Computer Engineering, Carnegie Mellon University, 5000 Forbes Ave., Pittsburgh, PA 15213. (e-mail: althoff@ece.cmu.edu).

Alexander Mergel is with the Institute of Automatic Control Engineering (LSR), Technische Universität München, 80290 München, Germany (e-mail: alexander.mergel@mytum.de).

Manuscript received xx. month 2010; revised xxx. situations are ignored. The majority of works on this topic use learning mechanisms such as neural networks and autoregressive exogenous models [37], [42], or filter techniques such as Kalman filters [26], [33]. Another line of research is to detect traffic participants on selected road sections and learn motion patterns for anomaly detection. Developed learning techniques for motion patterns are e.g. clustering methods [20], hidden Markov models [30], and growing hidden Markov models [39]. The disadvantage of a prediction at fixed locations is that the predictions are specialized to this particular road segment and are probably not representative for other traffic situations.

For the prediction of arbitrary traffic situations, simulations of traffic participants have been used [5], [18]. Due to the efficiency of single simulations, these approaches are already widely implemented in cars, e.g. to initiate an emergency braking maneuver based on measures like time to collision. Simulations of traffic participants are also computed in microscopic traffic simulations [28], [38]. However, single simulations do not consider uncertainties in the measurements and actions of other traffic participants, which may lead to unsatisfactory collision predictions [25].

A more sophisticated threat assessment considers multiple simulations of other vehicles, considering different initial states and changes in their inputs (steering angle and acceleration). Multiple simulations have been used in [22] to identify collision-free maneuvers for triggering emergency braking. If the simulations are randomly generated, one computes by a so-called Monte-Carlo method, which have been studied in [4], [7]-[9], [13] for the risk analysis of road traffic and in [6], [40] for the related topic of air traffic safety.

Another method to compute the probabilistic behaviors of traffic participants, is to abstract their behavior. One approach is to linearize the system dynamics and compute with Gaussian distributions since the distribution remains Gaussian after a linear transformation [24]. Several linearizations representing different operation modes, such as stopping, or accelerating, are computed in [17]. The operation modes are probabilistically switched and the switching is Markovian. Another approach is to abstract traffic participants by Markov chains as presented in the previous works [2], [3].

\section{A. Contributions}

The main contribution of this paper is to compare the Monte Carlo simulation in [4], [7]-[9], [13] with the Markov chain abstraction in [2], [3] according to their performance in the 
probabilistic prediction of traffic situations. However, in order to compare both techniques, an appropriate behavior model has to be found to generate acceleration commands. For the first time, the behavior models used in [7]-[9], [13] and [2], [3] are analyzed according to the autocorrelation and the average spectral density. In addition, the abstraction of Markov chains is computed via simulation techniques instead of reachability analysis as in [2], [3] for better accuracy. Accuracy has further been improved in the Markov chain approach by resolving an unnecessary over-approximation in the crash probability computation and by using a more accurate vehicle model. The efficiency of the Markov chain computations and the Monte Carlo simulations have been increased, too: the Markov chain computation has been sped up by reformulating it to a sparse matrix multiplication and applying on-the-fly cancellation of non-relevant probabilities. The Monte Carlo simulations have been sped up by computing with analytical solutions instead of numerical solvers.

\section{B. Organization}

In Sec. II we give a detailed problem description. The modeling of traffic participants for the prediction is presented in Sec. III. We introduce the Markov chain abstraction and the Monte Carlo simulation in Sec. IV and Sec. V, respectively. The probabilistic generation of acceleration commands is presented in Sec. VI. The remainder of the paper is about the assessment of the Markov chain abstraction and the Monte Carlo simulation. First, the results for the prediction of the probabilistic occupancy are compared in Sec. VII. Second, the predicted crash probabilities are compared in Sec. VIII.

\section{Problem Description}

In order to get a better idea of the addressed problem, the required information and the objectives are described below.

\section{A. Required Information}

The presented safety assessment requires the following information:

- the planned trajectory of the autonomous car,

- the geometric description of the relevant road sections,

- the position and geometry of static obstacles,

- the position, velocity, and classification of traffic participants (into cars, trucks, motorbikes, bicycles, and pedestrians).

Note that the gathering of this data is not subject of this work. The planned trajectory of the ego car is known since it is internally planned. The geometric description of the relevant road sections can be extracted from off-the-shelf navigation systems and fused with lane detection software. For lane detection, one usually uses LIDAR sensors and cameras [29]. The same sensors are generally used for the detection of static obstacles. The detection of traffic participants and the estimation of their position and velocity is a challenging task [10], [32]. Since this data is difficult to infer, the result of dynamic object detection is usually described by a probability distribution.

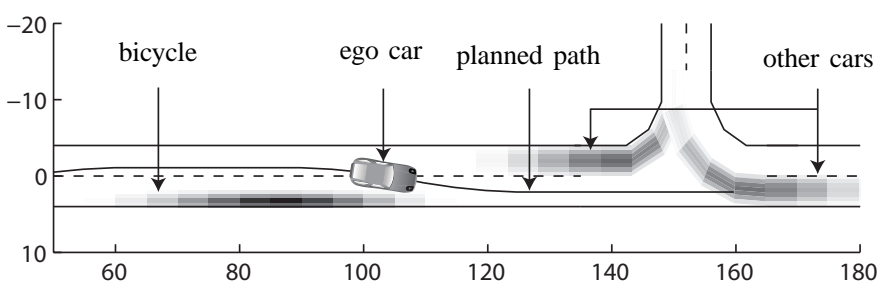

Fig. 1. Probabilistic occupancy of other traffic participants at a certain point in time. The result can be used for path planning of the ego car in order to avoid areas of high probability of occupancy.

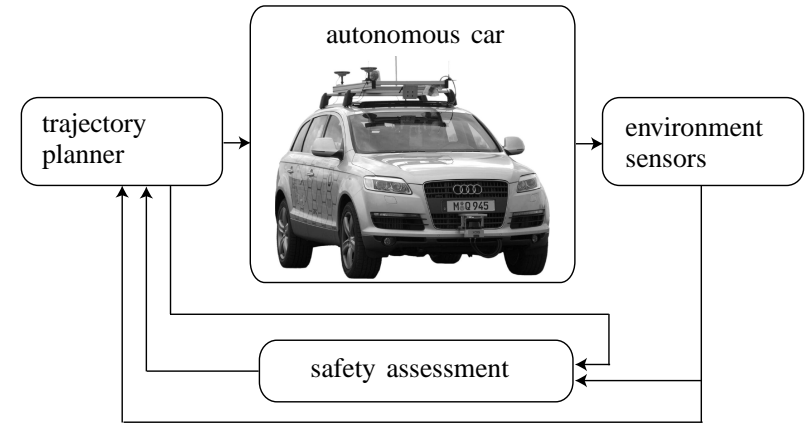

Fig. 2. Concept of the safety assessment: The environment sensors provide the road geometry, static obstacles, and traffic participants. This information is forwarded to the trajectory planner and the safety assessment module. The trajectory is only executed when approved by the safety assessment module.

The specialty of the proposed methods for the safety assessment is that they can compute with the uncertainties provided by the dynamic object detection. The probability distributions of measurements can be arbitrary and may have multiple maxima due to multiple object hypotheses. In addition to this uncertainty, there is another uncertainty regarding the future behavior of other traffic participants.

\section{B. Objectives}

Incorporating the uncertainty from the obstacle detection and the future behavior, this work predicts the future probabilistic occupancy of obstacles on the road. The second result of this work is the probability of a crash for the autonomous vehicle when following its planned path.

The probabilistic occupancy of other traffic participants allows to optimize the planned paths such that the autonomous car does not come to close to other traffic participants. This is illustrated in Fig. 1. The crash probability helps to decide if a planned trajectory should be executed or not. If several trajectories are planned, the safety assessment can identify the trajectory with the least crash probability. The interaction of the safety assessment module with the sensor information and the trajectory planner is illustrated in Fig. 2. In order to update the prediction for $t \in\left[0, t_{f}\right]$ after a time interval $\Delta t$ based on new sensor values, the computation has to be faster than realtime by a factor of $t_{f} / \Delta t$.

\section{Modeling of Traffic Participants}

This work focuses on the safety assessment of autonomous cars driving on a road network, i.e. the motion of traffic participants is constrained along designated roads. On that 
account, the motion of traffic participants is modeled such that traffic participants follow certain paths up to a certain accuracy. An alternative modeling for probabilistic prediction in unstructured environments can be found e.g. in [35].

The generated paths are located in the centers of lanes that can be followed. Possible lanes to be followed are extracted from detected road sections by checking all possible driving options, such as left turn, right turn, go straight, left lane change, and right lane change. Possible driving paths of an exemplary road section using the aforementioned driving options are shown in Fig. 3. In some traffic situations, additional paths which use lanes for oncoming traffic are required. This is the case when an obstacle (e.g. standing car) blocks a lane. Possible paths for such a scenario are shown in Fig. 4 and can be obtained with the same methods used in the trajectory planner, but applied to other traffic participants instead of the ego car. The concept of generating paths which are followed by a certain accuracy is also suggested in [17].

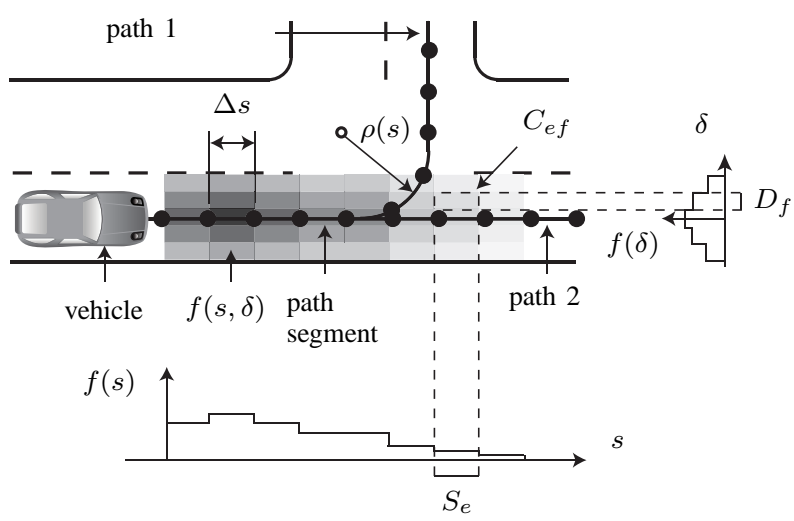

Fig. 3. Probability distribution of the position of a vehicle along a pathaligned coordinate system.

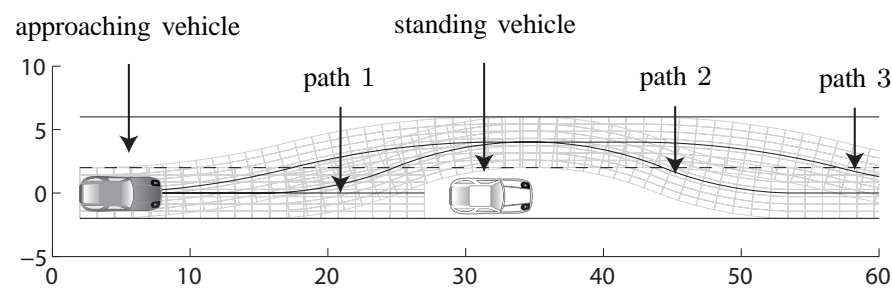

Fig. 4. Evasion of a standing car with alternative paths.

\section{A. Lateral Distribution}

The deviation along generated paths is modeled by a piecewise constant probability density function $f(\delta)$, where $\delta$ is the lateral deviation from a driving path as shown in Fig. 3 . The deviation probability of other traffic participants at the beginning of the prediction is set according to the measurement uncertainty of the obstacle detection. This distribution can be cross-faded over time to a distribution that has been obtained from an averaging of traffic observations; see [11].

\section{B. Longitudinal Distribution}

In contrast to the path deviation, the longitudinal dynamics of traffic participants along a path can be much better described by a mathematical model. In order to formulate this model, the volumetric center of traffic participants along a path is denoted by $s$, the velocity by $v$, and the absolute acceleration by $a$. The acceleration command $u$ is normalized and varies from $[-1,1]$, where -1 represents full braking and 1 represents full acceleration. Further, the function $\rho(s)$ is introduced which maps the path coordinate $s$ to the radius of curvature. The radius of the path determines the tangential acceleration $a_{T}$, and the normal acceleration is denoted by $a_{N}$. The differential equations for the vehicle dynamics are:

$$
\dot{s}=v, \quad \dot{v}=\left\{\begin{array}{l}
a^{\max } u, \quad 0<v \leq v^{\mathrm{sw}} \vee u \leq 0 \\
a^{\max } \frac{v^{\mathrm{sw}}}{v} u, \quad v>v^{\mathrm{sw}} \wedge u>0 \\
0, \quad v \leq 0
\end{array}\right.
$$

subject to the constraints

$$
\begin{aligned}
v & \leq v^{\max }, \\
|a| & \leq a^{\max }, \quad|a|=\sqrt{a_{N}^{2}+a_{T}^{2}}, \quad a_{N}=v^{2} / \rho(s), a_{T}=\dot{v} .
\end{aligned}
$$

Backwards driving on a lane is not considered; see (1) $(\dot{v}=0, v \leq 0)$. The positive acceleration dynamic changes at the switching velocity $v^{\mathrm{sw}}$. The dynamics for $0<v \leq$ $v^{\mathrm{sw}} \vee u \leq 0$ is based on tire friction, while the other one is based on engine power which limits the acceleration when the torque for $v>v^{\mathrm{sw}}$ is not causing wheel spin anymore. The constraint $v \leq v^{\max }$ in (2) models the speed limit. The other constraint models that the tire friction only allows a limited absolute acceleration $a^{\max }$ (Kamm's circle). The constant $a^{\max }$ is chosen as $a^{\max }=7\left[\mathrm{~m} / \mathrm{s}^{2}\right]$ and $v^{\mathrm{sw}}$ is chosen according to the different classes of traffic participants ${ }^{1}$.

The proposed model for the longitudinal dynamics is almost the same as used in [13]. The difference there is that the acceleration command resulting in zero acceleration is nonzero. It is also remarked that the model in this work differs from the previous one in [2]. The new model is more accurate which can be observed by plotting the acceleration over the velocity, and comparing it with the result of a high fidelity simulation $^{2}$ of an Audi Q7 in Fig. 5. Note that the peaks in acceleration occur due to the torque converter of the automatic gear box.

A specialty of the proposed longitudinal dynamics is that an analytical solution exists for constant $u$. This is beneficial for the Monte Carlo simulation, introduced later.

Proposition 1 (Analytical Solution of (1)): The analytical solution of the longitudinal dynamics of traffic participants in (1) for $u>0(u=\mathrm{const})$ and $v>v^{\mathrm{sw}}$ is

$$
\begin{aligned}
& s(t)=s(0)+\frac{\left(v(0)^{2}+2 v^{\mathrm{sw}} u t\right)^{\frac{3}{2}}-v(0)^{3}}{3 v^{\mathrm{sw}} u}, \\
& v(t)=\sqrt{v(0)^{2}+2 v^{\mathrm{sw}} u t} .
\end{aligned}
$$

The analytical solutions of the cases $0<v<v^{\mathrm{sw}} \vee u<0$ and $v \leq 0$ are trivial.

${ }^{1}$ Used values in this work: Car: $7.3[\mathrm{~m} / \mathrm{s}]$, truck: $4[\mathrm{~m} / \mathrm{s}]$, motorbike: $8[\mathrm{~m} / \mathrm{s}]$, bicycle: $1[\mathrm{~m} / \mathrm{s}]$.

${ }^{2}$ Used software: veDYNA. 


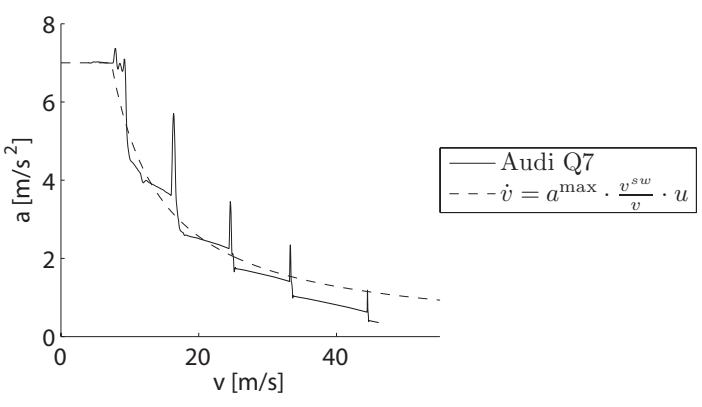

Fig. 5. Maximum acceleration $a$ of the Audi Q7 plotted over its velocity $v$; used parameters: $a^{\max }=7\left[\mathrm{~m} / \mathrm{s}^{2}\right], v^{\mathrm{sw}}=7.3[\mathrm{~m} / \mathrm{s}]$.

The correctness can be easily verified by inserting the solution into (1).

\section{Combined Distribution}

The driver task of velocity control and lane keeping can be assumed to be fairly independent; see [14], [21]. The same observation holds for autonomous vehicles whose vehicle control is separately developed for lateral and longitudinal dynamics [34], or whose control has negligible dependency with respect to the safety assessment in road traffic. Thus, it is assumed that the longitudinal probability distribution $f(s)$, obtained from the longitudinal dynamics (1), is independent from the lateral distribution so that the overall distribution is $f(s, \delta)=f(s) \cdot f(\delta)$ as indicated in Fig. 3. It is emphasized that the lateral and the longitudinal distribution $f(\delta)$ and $f(s)$ refer to the volumetric center of the vehicle bodies.

In this work, the lateral and the longitudinal probability distribution are modeled as a piecewise constant distribution as shown in Fig. 3. An interval with constant probability distribution along a path is denoted by $S_{e}$ and by $D_{f}$ for a deviation interval. The region with constant probability distribution spanned by $S_{e}$ and $D_{f}$ is denoted by $C_{e f}$ (see Fig. 3), which is required later.

The vehicle bodies and the occupancy of pedestrians on the road is modeled by rectangles whose size varies between the different types of traffic participants (cars, trucks, motorbikes/bicycles, and pedestrians).

\section{MARKOV Chain ABStRaCtion}

First, Markov chain abstraction is considered for predicting the longitudinal probability distribution. The techniques for the abstraction of dynamic systems with continuous state variables to Markov chains are manifold, where many of them couple the time increment with the accuracy of the abstraction, see e.g. [19], [23]. However, this coupling is unfavorable in terms of real-time applicability, as a required approximation accuracy may lead to short time increments of probability updates - and consequently to too many update iterations that cannot be handled in real-time. For this reason, the update intervals and the approximation accuracy is decoupled as in [27], [36].

The abstraction, which is computed offline, allows the dynamics of a traffic participant (1) to be described by a Markov chain. During online operation, a Markov chain is

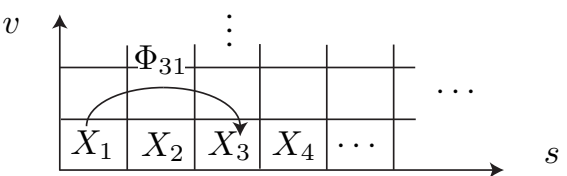

Fig. 6. Discretization of the state space.

instantiated for each relevant traffic participant around the ego vehicle.

A Markov chain is a stochastic dynamic system with discrete state $z \in \mathbb{N}^{+}$. There are discrete time and continuous time Markov chains. In this work, discrete time Markov chains are used, such that $t \in\left\{t_{1}, t_{2}, \ldots, t_{f}\right\}$ where $t_{f}$ is the prediction horizon and $t_{k+1}-t_{k}=T \in \mathbb{R}^{+}$is the time step increment. The current state of Markov chains is not exactly known, but probabilities $p_{i}=P(z=i)$ describe that the system is in state $z=i$, which are combined to a probability vector $p$ for all states. By definition, the probability vector for the next time step $t_{k+1}$ is a linear combination of the probability vector of the previous time step $t_{k}$ :

$$
p\left(t_{k+1}\right)=\Phi p\left(t_{k}\right),
$$

where $\Phi$ is referred to as the transition matrix.

Since the original system has continuous state variables, regions of the continuous state space have to be assigned to discrete states. In this work, a region $X \subset \mathbb{R}^{2}$ of the continuous state space of (1) is discretized in orthogonal cells of equal size, resulting in rectangular cells, see Fig. 6. The state space cells are denoted by $X_{i}$, where the index $i$ refers to the value of the corresponding discrete state. In an analogous way, the region $U=[-1,1]$ of the input space of $(1)$ is discretized into intervals $U^{\alpha}$. The index $\alpha$ refers to the value of the discrete input $y$. In order to distinguish between indices referring to discrete state or input values, state indices are subscripted and Latin, where input indices are superscripted and Greek. In order to obtain a continuous distribution from the probability vector $p$, it is assumed that the probability distribution is uniform within each cell.

\section{A. Transition Probabilities of the Markov Chain}

The transition probabilities store the probabilities that the discrete state changes from $j$ to $i$ : $\Phi_{i j}=P\left(z\left(t_{k+1}\right)=\right.$ $\left.i \mid z\left(t_{k}\right)=j\right)$. In this work, the transition probabilities depend on the value of the discrete input $y$, too. For this reason, a different transition probability matrix $\Phi^{\alpha}$ is computed for each discrete input value $\alpha$, such that $\Phi_{i j}^{\alpha}=P\left(z\left(t_{k+1}\right)=\right.$ $\left.i, y\left(t_{k+1}\right)=\alpha \mid z\left(t_{k}\right)=j, y\left(t_{k}\right)=\alpha\right)$.

The transition probabilities are obtained by running $N_{j}^{\alpha}$ simulations starting from the initial cell $X_{j}$ under input $u \in U^{\alpha}$. The number $N_{i j}^{\alpha}$ of those simulations ending up in cell $X_{i}$ determines the transition probability: $\Phi_{i j}^{\alpha}=N_{i j}^{\alpha} / N_{j}^{\alpha}$.

The input values are held constant during the simulation, but have varying values from simulation to simulation. Since it is assumed that the initial states and the inputs are uniformly distributed within cells, the initial states and the inputs are drawn from a uniform grid within the cells as illustrated in Fig. 7. 

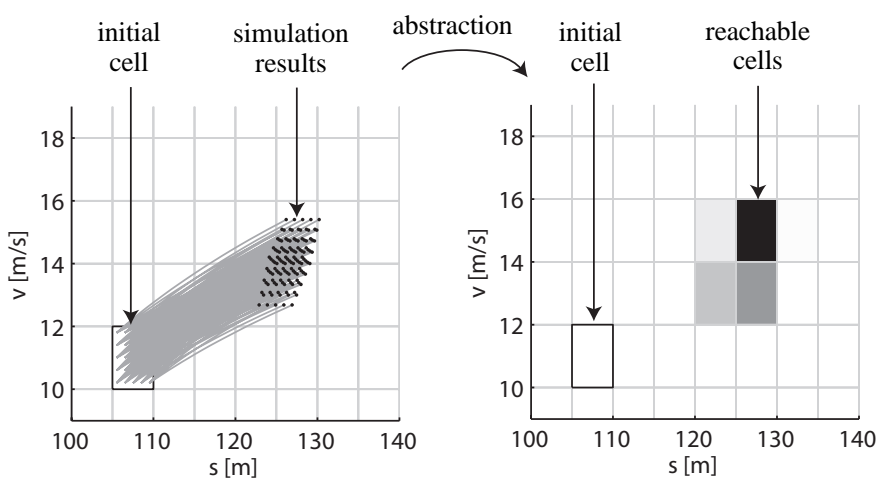

Fig. 7. Simulation results of the original system (left) and the corresponding probabilistic reachable set of the abstracting Markov chain (right). Both results are obtained using the same initial cell and input cell. A transition to a cell is the more likely, the darker the color of the cell is.

Another possibility to abstract a continuous dynamics to a Markov chain is via reachability analysis as described in [2]. This technique is less accurate in terms of the resulting transition probabilities, but makes it possible to compute complete abstractions, i.e. all states reachable by the abstracted Markov chain (cells with non-zero probability) cover all possible trajectories of the original system. Completeness makes it possible to guarantee that no crash occurs when the crash probability is 0 . However, a crash-free trajectory can also be guaranteed when the intersection between reachable road sections of traffic participants and the autonomous car are empty. Reachable road sections can be computed as proposed in [2]. Vehicles that do not pose a threat can be ignored for the probabilistic computations which is also proposed in [17]. Since completeness is not required, the more accurate simulation technique is applied.

\section{B. Computing Stochastic Reachable Sets using Markov Chains}

The transition probabilities allow to compute the state probabilities as shown in (3). The difference, however, is that the transition probabilities incorporate the input probability, too. In order to use the update scheme of a Markov chain, the state probability vector has to be redefined. Denoting the joint probability of a state value and an input value as $p_{i}^{\alpha}:=P(z=i, y=\alpha)$, the combined probability vector is defined as

$$
\tilde{p}^{T}=\left[\begin{array}{llllllllll}
p_{1}^{1} & p_{1}^{2} & \ldots & p_{1}^{c} & p_{2}^{1} & \ldots & p_{2}^{c} & p_{3}^{1} & \ldots & p_{d}^{c}
\end{array}\right],
$$

where $d$ is the number of states and $c$ is the number of inputs. Given this probability vector, the transition values have to be organized in the transition matrix as

$\tilde{\Phi}=\left[\begin{array}{ccccccccc}\Phi_{11}^{1} & 0 & \ldots & 0 & \ldots & \Phi_{1 d}^{1} & 0 & \ldots & 0 \\ 0 & \Phi_{11}^{2} & \ldots & 0 & \ldots & 0 & \Phi_{1 d}^{2} & \ldots & 0 \\ \vdots & & & & & & & \vdots & \\ 0 & 0 & \ldots & \Phi_{d 1}^{c} & \ldots & 0 & 0 & \ldots & \Phi_{d d}^{c}\end{array}\right]$

The rewritings allows to update the probabilities by $\tilde{p}\left(t_{k+1}\right)=\tilde{\Phi} \tilde{p}\left(t_{k}\right)$. The transition matrix $\tilde{\Phi}$ is sparse, i.e. it does not contain many non-zero values which allows to increase the efficiency of the matrix multiplication (see e.g. [43]). The efficiency is even more increased when canceling small probabilities in $\tilde{p}$ after each time step $t_{k}$ and normalizing $\tilde{p}$ afterwards such that its sum is one. This is reasonable since completeness is not required. The value $\underline{\tilde{p}}$ below which probabilities are canceled is set by the minimum probability density $\underline{\xi}$ which is uniform within each cell according to previous assumptions so that

$$
\underline{\tilde{p}}=s^{\Delta} v^{\Delta} u^{\Delta} \underline{\xi},
$$

where $s^{\Delta}, v^{\Delta}$, and $u^{\Delta}$ are the cell lengths of position, velocity, and input value. The default value of $\underline{\xi}$ is 0 (no cancelation), good results have been obtained with $\underline{\underline{\xi}}=1 / 16 \cdot 10^{-3}$. Higher values of $\underline{\xi}$ reduce computation time, while decreasing the accuracy.

\section{Monte Carlo Simulation}

There exists a huge variety of Monte Carlo methods and thus one cannot give a strict guidance on how to apply them in general. However, most methods exhibit the following scheme.

1) Generate inputs and initial states randomly.

2) Perform a deterministic computation starting at the initial states subject to the randomly generated inputs.

3) Aggregate the results of the individual computations into the final result.

In this work, the initial states and the input values have a piecewise constant probability distribution in order to compare the results with the Markov chain computations. The random values are obtained by the inverse transform method (see [41]) according to the given piecewise constant probability distributions. The deterministic computations for the longitudinal dynamics are simply computed as presented in Prop. 1. The aggregation of results is discussed in more detail below.

\section{A. Aggregation of Results}

The aggregation of the results depends on the purpose of the Monte Carlo simulation. For the computation of the probabilistic occupancy, it is checked in which segment (seg) $S_{i}$ of the followed path a simulation ends. For the computation of the crash probability, it is checked if a crash occurs. The detection of these events is formalized by indicator functions which use the general vector $x$ containing all variables of the Monte Carlo simulation:

$\operatorname{ind}_{i}^{\text {seg }}(x)=\left\{\begin{array}{l}1, \text { if } s(x) \in S_{i} \\ 0, \text { otherwise }\end{array}, \quad\right.$ ind $^{\text {crash }}(x)=\left\{\begin{array}{l}1, \text { crash } \\ 0, \text { otherw. }\end{array}\right.$

Since in this work, all simulations have equal weight, the probabilities of occupying a path region or causing a crash are obtained by the relative number of simulations for which the indicator function is 1 .

\section{B. Error Analysis}

An intrinsic property of Monte Carlo simulation is that the result of the computations is not deterministic, i.e. the result differs from execution to execution. Obviously, this is because the samples for the deterministic simulation are randomly 
generated. Thus, the probability distributions are possibly far from the exact solution. The good news, however, is that the mean error scales with $\frac{1}{\sqrt{N_{s}}}$ where $N_{s}$ is the number of simulations. This is a well known result from Monte Carlo integration [41] of probability density functions $f(x)$, and holds for the problems discussed herein. This can be shown by reformulating the computations as a Monte Carlo integration using the indicator functions. The probability $\hat{p}_{i}$ that the path position $s$ is in $S_{i}$, and the crash probability $p^{C}$, are computed as

$$
\hat{p}_{i}=\int \operatorname{ind}_{i}^{\operatorname{seg}}(x) f(x) d x, \quad p^{C}=\int \operatorname{ind}^{\operatorname{crash}}(x) f(x) d x .
$$

\section{Vi. Generation of Acceleration Commands}

An important influence on the outcome of the probabilistic predictions are given by the sequence of probabilistic acceleration commands over time. Two methods for the generation of acceleration commands are presented and then compared. The first one is a Markov chain approach as presented in [2]. The other one is an approach based on Monte Carlo simulation as presented in [8], [9], [13]. In all approaches, the input value is held constant for a certain time span. In this work, this time span is chosen as for the time increment $T$ of the Markov chain abstraction. The comparison of the generated acceleration commands to real world measurements is future work.

\section{A. Markov Chain Approach}

In [2], not only the state transitions, but also the probabilistic acceleration values are generated by Markov chains. As mentioned above, the acceleration commands are held constant during the time intervals $\left[t_{k}, t_{k+1}\right]$ and are changed instantly at times $t_{k}$. The probability that the input value changes for a given state value, is described by the input transition values $\Gamma_{i}^{\alpha \beta}\left(t_{k}\right):=P\left(z\left(t_{k}^{\prime}\right)=i, y\left(t_{k}^{\prime}\right)=\alpha \mid z\left(t_{k}\right)=i, y\left(t_{k}\right)=\beta\right)$, where $t_{k}^{\prime}=t_{k}+\delta t$ and $\delta t$ is an infinitesimal time step. Those transition values are combined into an input transition matrix $\tilde{\Gamma}\left(t_{k}\right)$, similarly as for the state transition matrix $\tilde{\Phi}$ :

$\tilde{\Gamma}=\left[\begin{array}{cccccccccc}\Gamma_{1}^{11} & \Gamma_{1}^{12} & \ldots & \Gamma_{1}^{1 c} & 0 & \ldots & 0 & 0 & \ldots & 0 \\ \Gamma_{1}^{21} & \Gamma_{1}^{22} & \ldots & \Gamma_{1}^{2 c} & 0 & \ldots & 0 & 0 & \ldots & 0 \\ \vdots & & & & & & & \vdots & & \vdots \\ 0 & 0 & \ldots & 0 & 0 & \ldots & 0 & \Gamma_{d}^{c 1} & \ldots & \Gamma_{d}^{c c}\end{array}\right]$

In contrast to the computation of the transition matrix $\tilde{\Phi}$ for the states, the transition matrices $\tilde{\Gamma}\left(t_{k}\right)$ for the input cannot be computed based on a reasonably simple dynamic model (due to complexity of human behavior or decision systems of autonomous vehicles). As a consequence, the transition matrices $\tilde{\Gamma}\left(t_{k}\right)$ have to be learned by observation or set by a combination of offline simulations and heuristics, where the latter is used in [2] considering the constraints of the vehicle model in (2).

Combining the input and the state transition matrix yields the extended formula of the Markov chain update for time varying input probabilities:

$$
\tilde{p}\left(t_{k+1}\right)=\tilde{\Gamma}\left(t_{k}\right) \tilde{\Phi} \tilde{p}\left(t_{k}\right) .
$$

\section{B. Trajectory Weighting}

Another way of creating random inputs is proposed in works on Monte Carlo simulation of road traffic scenes [8], [9], [13]. In these approaches, a random trajectory is created first, and afterwards its likeliness is evaluated, i.e. a weight is assigned to it. The values of the input trajectories are created by an IID $^{3}$ process, but the final process is no longer IID after a weight is assigned by the likeliness function. The inputs created in the aforementioned works are for the acceleration and the steering, while only the acceleration is of interest in this work. After removing the steering-related aspects, the socalled goal function for the computation of the likeliness of the input trajectory is computed in [9], [13] as

$g(u)=-\sum_{k=1}^{N_{u}}\left(\lambda_{1}\left(v\left(t_{k}\right)-v^{\max }\right)^{2}+\lambda_{2} a_{T}\left(t_{k}\right)^{2}+\lambda_{3} a_{N}\left(t_{k}\right)^{2}\right)$

where $\lambda_{1}-\lambda_{3}$ are tuning parameters which punish velocity deviations from the allowed velocity, and large normal as well as tangential accelerations. The probability distribution of the input trajectories $f(u)$ is assumed to be $f(u)=c_{n} \exp \left(k_{g}\right.$. $g(u)$ ), where the previously introduced goal function is in the exponent. The value $k_{g}$ is another tuning parameter and $c_{n}$ is the normalization constant.

\section{Comparison}

Continuous input values generated from the Markov chain approach and the trajectory weighting approach are compared according to their autocorrelation and their average spectral density. Continuous input values are obtained from the discrete inputs of the Markov chain approach by the previously mentioned inverse transform method. The input trajectories for both approaches are generated with parameters listed in Tab. I for $N_{u}=10$, where $N_{u}$ is the number of time steps. The Monte Carlo simulation parameters are taken from [8]. The initial velocity is uniformly distributed within $v(0)=15 \pm 1 \mathrm{~m} / \mathrm{s}$, which affects the input generation due to the speed limit. The considered time horizon is $t_{f}=5 \mathrm{~s}$, and the number of computed simulations is $N_{s}=10^{5}$.

TABLE I

PARAMETERS FOR INPUT TRAJECTORY GENERATION.

\begin{tabular}{|ll|}
\hline General \\
\hline$v^{\max }$ & $100 / 3.6 \mathrm{~m} / \mathrm{s}$ \\
$T$ & $0.5 \mathrm{~s}$ \\
\hline \multicolumn{2}{|l|}{ Trajectory weighting; see [13] } \\
\hline$k_{g}$ & 100 \\
$\lambda_{1}$ & $0.05 / N_{u} /\left(1+v(0)^{2}\right)$ \\
$\lambda_{2}$ & $0.05 / N_{u} /\left(a^{\max }\right)^{2}$ \\
$\lambda_{3}$ & $0.05 / N_{u} /\left(a^{\max }\right)^{2}$ \\
\hline Markov chain; see $[2]$ \\
\hline$m$ & {$[0.01,0.04,0.25,0.25,0.4,0.05]$} \\
$q(0)$ & {$[0,0,0.5,0.5,0,0]$} \\
$\gamma$ & 0.2 \\
\hline
\end{tabular}

${ }^{3}$ IID: independent and identically distributed. 
1) Autocorrelation: The autocorrelation, i.e. the correlation of the signal against a time-shifted version of itself is defined as

$$
S(t, \tau):=E[\mathbf{u}(t) \mathbf{u}(\tau)]=\int_{-\infty}^{\infty} \int_{-\infty}^{\infty} u_{t} u_{\tau} f\left(u_{t} u_{\tau}\right) d u_{t} d u_{\tau},
$$

where $E[]$ is the expectation, $f()$ is the probability density function, and $u_{t}$ is a realization of the random variable $\mathbf{u}(t)$. The computation of the above integrals is approximated using Monte Carlo integration.

The autocorrelation values for the input trajectories are plotted in Fig. 8. There is almost no correlation between the inputs of different time steps in the trajectory weighting approach as shown in Fig. 8(a). This is in contrast to the Markov chain approach, where the input signals are much more correlated; see Fig. 8(b).

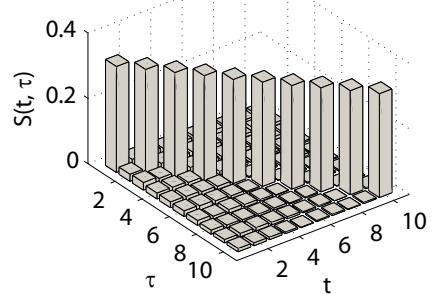

(a) Trajectory weighting approach.

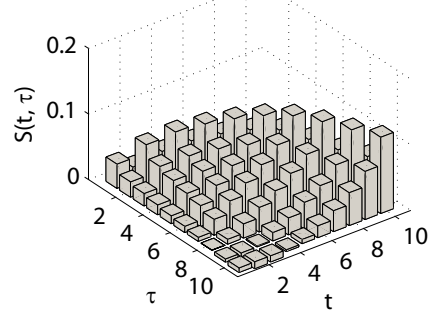

(b) Markov chain approach.
Fig. 8. Autocorrelation of input trajectories.

2) Average Spectral Density: Besides the autocorrelation, the spectral density of input signals is compared for a deeper analysis. The spectral density describes how the energy of a signal is distributed over its frequency, where the energy of a signal $x(t)$ is defined as $\int_{-\infty}^{\infty}|x(t)|^{2} d t$ in signal processing. The spectral density is defined as $|X(f)|^{2}$, where $X(f)$ is the Fourier transform of $x(t)$. For the analysis of several instances of a stochastic signal, one is interested in the expectation of the random spectral density $E\left[|\mathbf{X}(f)|^{2}\right]$.

Proposition 2 (Average Spectral Density): The average of the spectral density $\Phi(f)=E\left[|\mathbf{U}(f)|^{2}\right]$ for input signals $u(t)$, which are piecewise constant for time increments $T$, is computed as

$$
\Phi(f)=T^{2} \mathbf{s i}^{2}(\pi f T) \sum_{k=1}^{N_{u}} \sum_{l=1}^{N_{u}} E\left[\mathbf{u}\left(t_{k}\right) \mathbf{u}\left(t_{l}\right)\right] e^{-j 2 \pi f T(k-l)},
$$

where $N_{u}$ is the number of time steps and $\operatorname{si}(x)=\sin (x) / x$ is the sinc function.

Proof:

The input signal $u(t)$ is constant within consecutive time intervals $\left[t_{k}, t_{k+1}\right]$, where $t_{k+1}-t_{k}=T$. This can be written as

$$
u(t)=\sum_{k=1}^{N_{u}} u\left(t_{k}\right) \operatorname{rect}\left(\frac{t-(k-0.5) T}{T}\right),
$$

where $\operatorname{rect}(t)$ is 1 for $-0.5<t<0.5$ and 0 otherwise.
The Fourier transform makes it possible to formulate the following relation between the time and frequency domain:

$$
\operatorname{rect}\left(\frac{t-(k-0.5) T}{T}\right) \multimap j T \operatorname{si}(\pi f T) e^{-j 2 \pi f(k-0.5) T} \text {. }
$$

For the whole input signal, the Fourier transform is $U(f)=$ $j T \operatorname{si}(\pi f T) e^{j \pi f T} \sum_{k=1}^{N_{u}} u\left(t_{k}\right) e^{-j 2 \pi f k T}$ from which follows the spectral density $\Phi(f)$.

The expectation $E\left[\mathbf{u}\left(t_{k}\right) \mathbf{u}\left(t_{l}\right)\right]$ is obtained from the autocorrelation in (6). The expectations of spectral densities $\Phi(f)$ are visualized in Fig. 9. It can be seen that the lower frequencies are more dominant in the Markov chain approach, while the frequency distribution of the trajectory weighting approach is close to an IID process with uniform distribution.

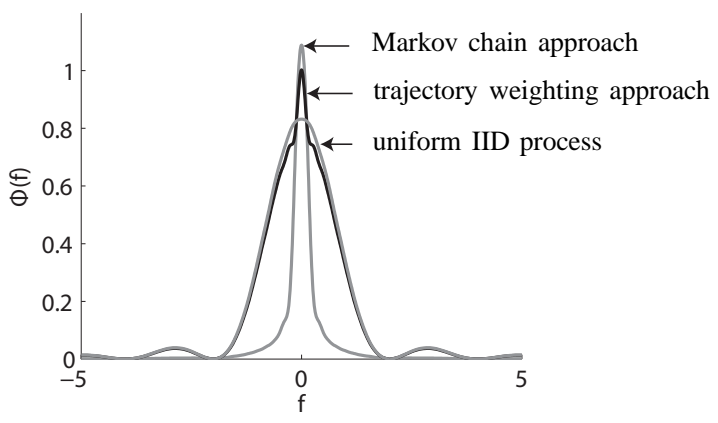

Fig. 9. Average spectral density of input trajectories.

A general observation in road traffic is that drivers change their acceleration with low frequency and that their inputs are highly correlated. In both tests, the autocorrelation and the average spectral density test, the acceleration inputs created by the Markov chain showed more realistic behavior.

\section{COMPARISON OF PRobabilistic OCCUPANCY}

In this section, acceleration commands from the previously presented Monte Carlo and Markov chain approach are used to compare the probability distribution of traffic participants. For each comparison, the same model for generating the acceleration commands is used, which ensures comparability of the distributions. Since the final probabilistic occupancy is simply obtained by $f(s, \delta)=f(s) \cdot f(\delta)$, where $f(\delta)$ is heuristically obtained, the following comparison is about $f(s)$ and the distribution of the velocity $f(v)$ as an auxiliary result.

The computed piecewise constant probability distributions of $f(s)$ and $f(v)$ with probabilities $p_{i}$ for certain intervals, are compared by the distance

$$
d=\sum_{i}\left|p_{i}-p_{i}^{\mathrm{e}}\right| V\left(X_{i}\right),
$$

where $p^{\mathrm{e}}$ is the exact probability distribution. The multiplication with the volume $V\left(X_{i}\right)$ of the cells $X_{i}$ is required in order to compare results of different discretization.

For the numerical experiments, two different Markov chain discretizations are used as listed in Tab. II. The cancelation of small probability values is performed as suggested in (4) with $\underline{\xi}=1 / 16 \cdot 10^{-3}$. The Monte Carlo approach used in the numerical experiment is performed with $10^{4}$ simulations. Since 
TABLE II

STATE SPACE DISCRETIZATIONS FOR A POSITION INTERVAL OF $[0,400] \mathrm{M}$ AND A VELOCITY INTERVAL OF $[0,60] \mathrm{M} / \mathrm{s}$.

\begin{tabular}{|lllll|}
\hline discretization & $\begin{array}{l}\text { position } \\
\text { segments }\end{array}$ & $\begin{array}{l}\text { position } \\
\text { resolution }\end{array}$ & $\begin{array}{l}\text { velocity } \\
\text { segments }\end{array}$ & $\begin{array}{l}\text { velocity } \\
\text { resolution }\end{array}$ \\
\hline $\mathrm{A}$ & 80 & $5 \mathrm{~m}$ & 30 & $2 \mathrm{~m} / \mathrm{s}$ \\
$\mathrm{B}$ & 320 & $1.25 \mathrm{~m}$ & 120 & $0.5 \mathrm{~m} / \mathrm{s}$ \\
\hline
\end{tabular}

there is no exact solution for the presented scenario, an almost exact solution was computed with Monte Carlo simulation using $10^{7}$ samples. The acceleration command is generated by a Markov chain using the parameters in Tab. I. The initial position and the initial velocity are uniformly distributed in the intervals $[2,8] \mathrm{m}$ and $[15,17] \mathrm{m} / \mathrm{s}$, respectively. The probability distributions are compared at $t=5 \mathrm{~s}$.

The resulting position and velocity distribution for different discretizations and inputs can be found in Fig. 10 and 11. The distance measure $d$ for all tested combinations of input generations, prediction techniques, and discretizations is listed in Tab. III. Since the Monte Carlo approach delivers different results for each run, 100 runs have been computed and the minimum, maximum, and mean value are shown. The results are fairly independent from the input generation, except that the Markov chain approach performs better when Monte Carlo generated inputs are used. This is because the probability of low velocities is high for those inputs so that the Markov chain specific problem of flattening distributions is limited to high velocities. The computational times can be found in Tab. IV, which are obtained from an AMD Athlon64 3700+ processor (single core) using a Matlab implementation. The Monte Carlo simulation has been obtained using the Runge-Kutta solver and the analytic solution as presented in Prop. 1. Ultimately, the Markov chain solution is faster than the analytically obtained Monte Carlo solution and the discretization of the Markov chain $B$ is fine enough to produce results that are more accurate than the Monte Carlo approach with $10^{4}$ simulations.

TABLE III

DISTANCE MEASURE $d$.

\begin{tabular}{|c|c|c|c|c|c|}
\hline & & $\begin{array}{l}\text { res. A } \\
\text { position }\end{array}$ & $\begin{array}{l}\text { res. A } \\
\text { velocity }\end{array}$ & $\begin{array}{l}\text { res. B } \\
\text { position }\end{array}$ & $\begin{array}{l}\text { res. B } \\
\text { velocity }\end{array}$ \\
\hline \multicolumn{6}{|c|}{ Input generation: Markov chain } \\
\hline \multirow[t]{3}{*}{ Monte Carlo: } & $\min$ & 0.0594 & 0.0218 & 0.0500 & 0.0166 \\
\hline & $\max$ & 0.2393 & 0.0783 & 0.0905 & 0.0331 \\
\hline & mean & 0.1327 & 0.0512 & 0.0677 & 0.0259 \\
\hline Markov chain: & & 1.0882 & 0.3425 & 0.0346 & 0.0121 \\
\hline \multicolumn{6}{|c|}{ Input generation: Monte Carlo } \\
\hline \multirow[t]{3}{*}{ Monte Carlo: } & $\min$ & 0.0885 & 0.0212 & 0.0573 & 0.0186 \\
\hline & $\max$ & 0.2169 & 0.0985 & 0.0936 & 0.0338 \\
\hline & mean & 0.1485 & 0.0518 & 0.0752 & 0.0261 \\
\hline Markov chain: & & 0.8438 & 0.1272 & 0.0165 & 0.0056 \\
\hline
\end{tabular}

TABLE IV

COMPUTATIONAL TIMES OF THE ROAD FOLLOWING SCENARIO.

\begin{tabular}{|llll|}
\hline $\begin{array}{l}\text { Monte Carlo } \\
\text { (simulated) }\end{array}$ & $\begin{array}{l}\text { Monte Carlo } \\
\text { (analytical) }\end{array}$ & Markov chain $A$ & Markov chain $B$ \\
\hline $3.44 \mathrm{~s}$ & $0.578 \mathrm{~s}$ & $0.030 \mathrm{~s}$ & $0.417 \mathrm{~s}$ \\
\hline
\end{tabular}
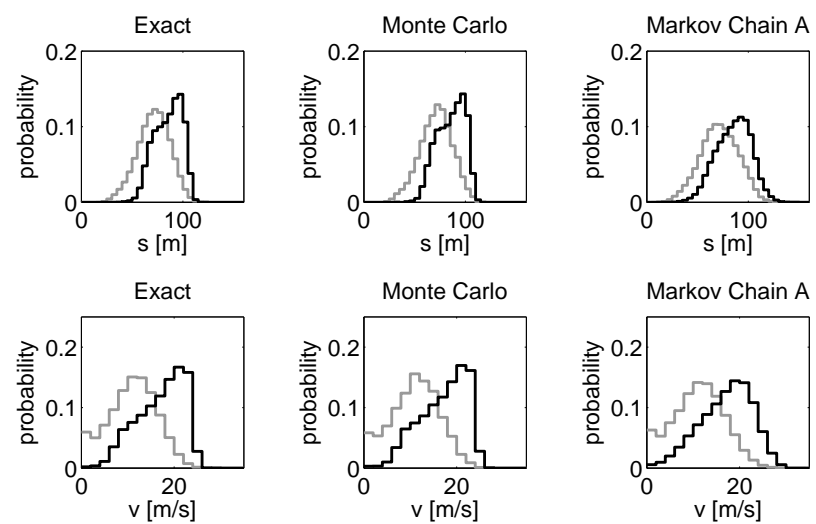

Fig. 10. Road following scenario: Position and velocity distribution for a coarse discretization $(t=5 \mathrm{~s}$ ). Inputs generated by the Markov chain approach result in the black distribution, while Monte Carlo generated inputs result in the gray distribution.
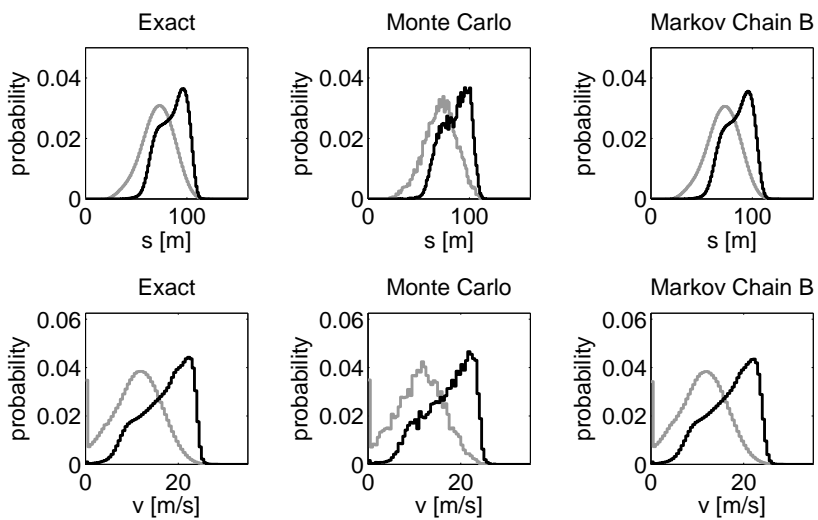

Fig. 11. Road following scenario: Position and velocity distribution for a fine discretization $(t=5 \mathrm{~s}$ ). Inputs generated by the Markov chain approach result in the black distribution, while Monte Carlo generated inputs result in the gray distribution.

Finally, it was analyzed if the quality of the probability distributions depends on the initial condition. As the vehicle model (1) is invariant under translations in position, it is only necessary to vary the initial velocity. The influence on the initial velocity on the distances $d^{\text {pos }}$, $d^{v e l}$ of the position and velocity is shown in Fig. 12. The Monte Carlo simulations are performed with $10^{4}$ samples and the Markov chain approach was computed with the $B$ model. In contrast to the previous computations, the speed limit of $100 / 3.6 \mathrm{~m} / \mathrm{s}$ has been removed so that initial velocities above this speed can be investigated. It can be seen that the dependence on the initial velocity and thus on the initial state can be neglected, meaning that the results in Fig. 10 and Fig. 11 are representative. The small dependence on the initial state makes it possible to tune the discretization based on a single initial distribution. If the distance measure $d$ of the position or velocity is too high, one can make the corresponding discretization finer until the desired accuracy is achieved, which should approximately hold for all other conditions.

\section{COMPARISON OF CRASh PROBABILITIES}

In this work, the crash probability of different time intervals is computed independently of previous crash probabilities. 


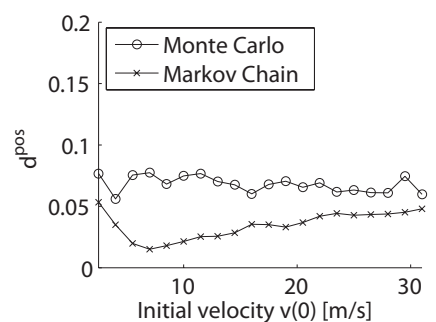

(a) Distance $d$ of the position distri- (b) Distance $d$ of the velocity distribution. bution.

Fig. 12. Distance $d$ to the exact solution for different initial velocities.

This has the advantage that for each time interval, a situation can be judged independently of previous occurrences. This is not the case, when computing the physical probability that a crash will happen. Consider a scenario in which two situations are equally dangerous at two different points in time. However, the probability that the vehicle crashes in the first situation is greater than in the second situation. This is because a crash can only occur in the second situation, if the vehicle survived the first one and crashes in the second one. For this reason, it is assumed that the autonomous vehicle has not crashed until the investigated time interval.

The crash probability is computed for consecutive time intervals, since one may miss a high crash probability when only computing for points in time. This is achieved in the Monte Carlo simulation by computing for sufficient intermediate points in time within a time interval. When using Markov chains, an additional transition matrix for time intervals is computed offline: $\tilde{\Phi}^{\text {interval }}=\frac{1}{\tilde{n}} \sum_{k=1}^{\tilde{n}} \tilde{\Phi}^{\left(\tilde{t}_{k}\right)}$, where $\tilde{\Phi}^{\left(\tilde{t}_{k}\right)}$ are transition probability matrices for intermediate points in time $\tilde{t}_{k}$. The probability distribution for time intervals is then computed as $\tilde{p}\left(t_{k}\right)^{\text {interval }}=\tilde{\Phi}^{\text {interval }} \tilde{p}\left(t_{k}\right)$, where $\tilde{p}\left(t_{k}\right)$ is computed in (5).

\section{A. Computation of Crash Probabilities}

The computation of crash probabilities is separately discussed for the Markov chain abstraction and the Monte Carlo simulation.

1) Markov Chain Abstraction: When using the Markov chain abstraction, one has to compute the probabilistic occupancy as an intermediate step. In order to describe the probabilistic occupancy, some additional notations have to be introduced. The region spanned by the path position interval $S_{e}$ and the deviation interval $D_{f}$ is denoted by $C_{e f}$ (see Fig. 3). The probability that the center of a vehicle is in a region $C_{e f}$ is denoted by $\hat{p}_{e f}$. The probabilities for the autonomous car are indicated by the superscript ego (e.g. $\left.\hat{p}_{g h}^{\text {ego }}\right)$. It is required to additionally introduce the probability $p_{\text {ghef }}^{\text {int }}$ that two bodies of the ego vehicle and another vehicle intersect when the center of the ego vehicle body is in $C_{g h}^{\text {ego }}$ and that of the other vehicle in $C_{e f}$. This probability is computed by gridding the regions $C_{g h}^{\text {ego }}, C_{e f}$ and testing for all combinations of centers if an intersection of vehicle bodies exists. The relative number of intersections provides the intersection probability. This computation is expensive, so that the intersection probabilities are precomputed offline by storing the results for different relative positions, orientations, and types of traffic participants in a database. In the previous work [2], $p_{\text {ght }}^{\text {int }}$ was conservatively chosen to 1 when an intersection is possible.

Using the introduced variables, the crash probability between the autonomous vehicle and another vehicle can be formulated as

$$
p^{C}=\sum_{g, h, e, f} p_{g h e f}^{\text {int }} \hat{p}_{g h}^{\text {ego }} \hat{p}_{e f} .
$$

The summation over all possible indices $g, h, e, f$ is computationally expensive. For this reason, techniques to effectively search for combinations of path and deviation indices which potentially cause a vehicle body intersection, are presented in the previous work [2].

2) Monte Carlo Simulation: The crash probability for the Monte Carlo approach is simply obtained by summing up the probabilities of simulations that have crashed. Note that simulations resulting in a crash are not removed from the computation in order to obtain crash probabilities in compliance with the aforementioned definition of the crash probability.

It is crucial that the detection of a crash is computationally cheap. Crashes are detected by checking if the rectangular vehicle bodies intersect. An efficient method to detect the intersection of two rectangles, is the separating axis theorem [16]. An extension considering the velocity of objects is presented in [12].

\section{B. Crash Scenario}

The crash probabilities are investigated for a scenario where the autonomous car drives behind another car in the same lane. The autonomous car starts from the position $0 \mathrm{~m}$ with constant velocity $20 \mathrm{~m} / \mathrm{s}$ and has a uniform position uncertainty of $\pm 3 \mathrm{~m}$. The vehicle driving in front has a uniform position uncertainty of $[20,25] \mathrm{m}$ and the initial velocity is within $[15,17] \mathrm{m} / \mathrm{s}$. The other parameters are as listed in Tab. I, and the considered time horizon is $t_{f}=5 \mathrm{~s}$. The (almost) exact solution is obtained from a Monte Carlo simulation with $10^{5}$ simulations.

The crash probability of the Markov chain approach is compared to the exact solution with a coarse and a fine discretization using model $A$ and $B$ (see Tab. II) and for points in time (TP) as well as time intervals (TI). The crash probabilities $p^{C}$ for different time steps/time intervals are shown in Fig. 13. It can be observed that the fine discretization produces much better results than the coarse discretization.

Besides different Markov chain models, Monte Carlo solutions were tested for a varying number of samples; see Fig. 13(c). The results show that the crash probability is very accurate, even when only $10^{3}$ or $10^{2}$ samples are used. For this reason, it can be clearly stated that the Monte Carlo simulation performs better than the Markov chain approach when the crash probability has to be computed. This is reconfirmed by the computational times in Tab. V, where the Monte Carlo approach is more efficient. The computational times for the Markov chain approach are separated into the part for computing the probability distribution and the part that intersects the probability distributions to obtain the crash probability. The 
computations were performed on an AMD Athlon64 3700+ processor (single core) using a Matlab implementation.

TABLE V

COMPUTATIONAL TIMES OF THE CRASH SCENARIO.

\begin{tabular}{|c|c|c|c|c|}
\hline \multicolumn{5}{|c|}{ Markov chain } \\
\hline & $A(\mathrm{TP})$ & $A(\mathrm{TI})$ & $B(\mathrm{TP})$ & $B(\mathrm{TI})$ \\
\hline Prob. dist. & $0.175 \mathrm{~s}$ & $0.175 \mathrm{~s}$ & $0.525 \mathrm{~s}$ & $0.525 \mathrm{~s}$ \\
\hline Intersection & $0.042 \mathrm{~s}$ & $0.107 \mathrm{~s}$ & $0.169 \mathrm{~s}$ & $0.394 \mathrm{~s}$ \\
\hline Total & $0.217 \mathrm{~s}$ & $0.282 \mathrm{~s}$ & $0.694 \mathrm{~s}$ & $0.919 \mathrm{~s}$ \\
\hline \multicolumn{5}{|c|}{ Monte Carlo simulation } \\
\hline & $1 \mathrm{e} 2(\operatorname{sim})$. & $1 \mathrm{e} 3(\operatorname{sim})$. & 1e2 (analy.) & 1e3 (analy.) \\
\hline Total & $0.190 \mathrm{~s}$ & $0.549 \mathrm{~s}$ & $0.069 \mathrm{~s}$ & $0.321 \mathrm{~s}$ \\
\hline
\end{tabular}
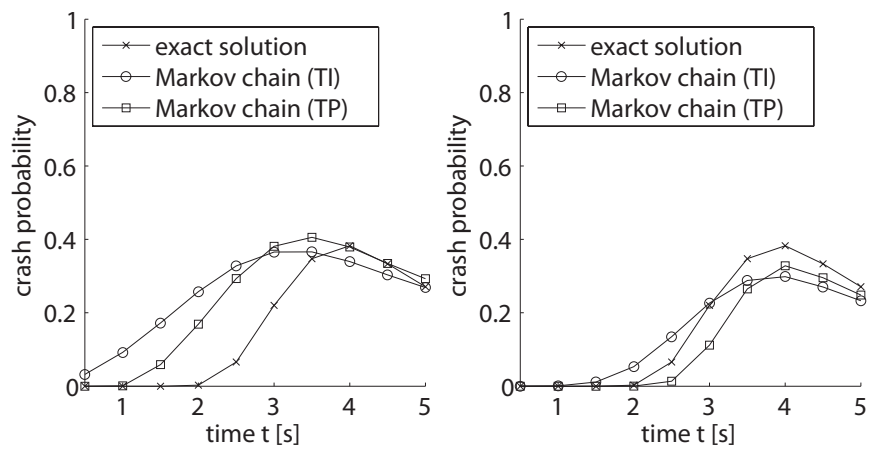

(a) Markov chain comparison (dis- (b) Markov chain comparison (discretization A). cretization B).

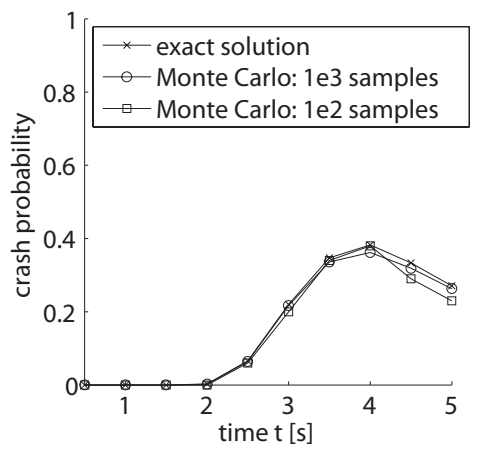

(c) Monte Carlo comparison.

Fig. 13. Crash probabilities for different points in time.

\section{COnclusions}

The Markov chain approach and the Monte Carlo approach have some inherent differences concerning their error sources. The main error in the Markov chain approach is introduced due to the discretization of the state and input space. The error in the transition probabilities can be made arbitrarily small since they are computed beforehand. Consequently, the Markov chain approach has only systematic errors from the discretization but no probabilistic errors since no random sampling is applied. Thus, the resulting probabilities are computed deterministically so that the results can be repeated.

In the Monte Carlo approach, there are no systematic errors (no bias) because each simulation is correctly solved with the original dynamical system equations. However, the Monte Carlo simulation suffers under probabilistic errors due to the sampling of the initial conditions and the input sequences. Due to the probabilistic errors, the resulting distributions and crash probabilities differ from execution to execution under unchanged initial conditions. This implies that the obtained results might be far off the exact solution - however, the likeliness of an extremely bad result is small and the mean error converges with $\frac{1}{\sqrt{N_{s}}}$, where $N_{s}$ is the number of samples.

The resulting probability distributions of the Markov chain approach are slightly more accurate and faster than for the Monte Carlo simulation if an analytical solution exists. When no analytical solution exists, the Markov chain approach is at least about 10 times faster. Because there are many matrix multiplications in the Markov chain approach, it can be significantly accelerated by using dedicated hardware such as DSPs (digital signal processors). However, when computing crash probabilities, the Monte Carlo approach clearly returns better results since it does not suffer from the discretization of the state space.

The results can be directly implemented in an autonomous car. A screenshot of the probabilistic prediction in the test vehicle MUCCI [15] is shown in Fig. 14.

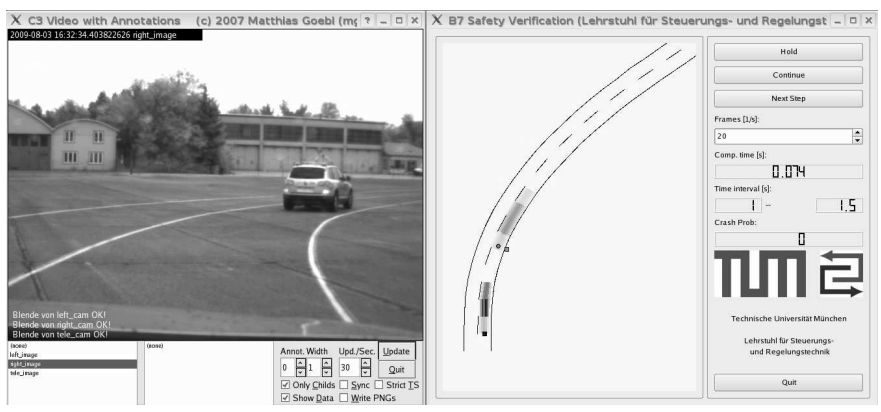

Fig. 14. Screenshot of a test drive. The probabilistic occupancy of the other car is computed via the Markov chain abstraction.

\section{ACKNOWLEDGMENT}

The authors gratefully acknowledge the partial financial support of this work by the Deutsche Forschungsgemeinschaft (German Research Foundation) within the Transregional Collaborative Research Center 28 "Cognitive Automobiles".

\section{REFERENCES}

[1] M. Abdel-Aty and A. Pande. ATMS implementation system for identifying traffic conditions leading to potential crashes. IEEE Transactions on Intelligent Transportation Systems, 7(1):78-91, 2006.

[2] M. Althoff, O. Stursberg, and M. Buss. Model-based probabilistic collision detection in autonomous driving. IEEE Transactions on Intelligent Transportation Systems, 10:299 - 310, 2009.

[3] M. Althoff, O. Stursberg, and M. Buss. Safety assessment of driving behavior in multi-lane traffic for autonomous vehicles. In Proc. of the IEEE Intelligent Vehicles Symposium, pages 893-900, 2009.

[4] K. Aso and T. Kindo. Stochastic decision-making method for autonomous driving system that minimizes collision probability. In Proc. of the FISITA World Automotive Congress, 2008.

[5] A. Barth and U. Franke. Estimating the driving state of oncoming vehicles from a moving platform using stereo vision. IEEE Transactions on Intelligent Transportation Systems, 10:560-571, 2009.

[6] H. A. P. Blom, J. Krystul, and G. J. Bakker. Free Flight Collision Risk Estimation by Sequential Monte Carlo Simulation, chapter 10, pages 247-279. Taylor \& Francis CRC Press, 2006. 
[7] A. E. Broadhurst, S. Baker, and T. Kanade. A prediction and planning framework for road safety analysis, obstacle avoidance and driver information. In Proc. of the 11th World Congress on Intelligent Transportation Systems, October 2004.

[8] A. E. Broadhurst, S. Baker, and T. Kanade. Monte Carlo road safety reasoning. In Proc. of the IEEE Intelligent Vehicles Symposium, pages 319-324, 2005.

[9] S. Danielsson, L. Petersson, and A. Eidehall. Monte Carlo based threat assessment: Analysis and improvements. In Proc. of the IEEE Intelligent Vehicles Symposium, pages 233-238, 2007.

[10] M. S. Darms, P. E. Rybski, C. Baker, and C. Urmson. Obstacle detection and tracking for the urban challenge. IEEE Transactions on Intelligent Transportation Systems, 10:475-485, 2009.

[11] P. P. Dey, S. Chandra, and S. Gangopadhaya. Lateral distribution of mixed traffic on two-lane roads. Journal of Transportation Engineering, 132:597-600, 2006.

[12] D. Eberly. Dynamic collision detection using oriented bounding boxes Technical report, Geometric Tools, Inc., 2002.

[13] A. Eidehall and L. Petersson. Statistical threat assessment for general road scenes using Monte Carlo sampling. IEEE Transactions on Intelligent Transportation Systems, 9:137-147, 2008.

[14] M. Gabibulayev and B. Ravani. A stochastic form of a human driver steering dynamics model. Journal of Dynamic Systems, Measurement, and Control, 129:322-336, 2007.

[15] M. Goebl, M. Althoff, M. Buss, G. Färber, F. Hecker, B. Heißing, S. Kraus, R. Nagel, F. Puente León, F. Rattei, M. Russ, M. Schweitzer, M. Thuy, C. Wang, and H.-J. Wünsche. Design and capabilities of the Munich cognitive automobile. In Proc. of the IEEE Intelligent Vehicles Symposium, pages 1101-1107, 2008.

[16] S. Gottschalk, M. C. Lin, and D. Manocha. OBBTree: A hierarchical structure for rapid interference detection. Computer Graphics, 30:171180,1996

[17] D. H. Greene, J. J. Liu, J. E. Reich, Y. Hirokawa, T. Mikami, H. Ito, and A. Shinagawa. A computationally-efficient collision early warning system for vehicles, pedestrian and bicyclists. In Proc. of the 15th World Congress on Intelligent Transportation Systems, 2008.

[18] J. Hillenbrand, A. M. Spieker, and K. Kroschel. A multilevel collision mitigation approach - its situation assessment, decision making, and performance tradeoffs. IEEE Transactions on Intelligent Transportation Systems, 7:528-540, 2006.

[19] J. Hu, M. Prandini, and S. Sastry. Aircraft conflict detection in presence of a spatially correlated wind field. IEEE Transactions on Intelligent Transportation Systems, 6:326-340, 2005.

[20] W. Hu, X. Xiao, Z. Fu, D. Xie, T. Tan, and S. Maybank. A system for learning statistical motion patterns. IEEE Transactions on Pattern Analysis and Machine Intelligence, 28:1450-1464, 2006.

[21] T. Jürgensohn. Control theory models of the driver. In P. C. Cacciabue, editor, Modelling Driver Behaviour in Automotive Environments, pages 277-292. Springer, 2007.

[22] N. Kaempchen, B. Schiele, and K. Dietmayer. Situation assessment of an autonomous emergency brake for arbitrary vehicle-to-vehicle collision scenarios. IEEE Transactions on Intelligent Transportation Systems, 10:678-687, 2009.

[23] X. Koutsoukos and D. Riley. Computational methods for reachability analysis of stochastic hybrid systems. In Hybrid Systems: Computation and Control, LNCS 3927, pages 377-391. Springer, 2006.

[24] A. Lambert, D. Gruyer, G. S. Pierre, and A. N. Ndjeng. Collision probability assessment for speed control. In Proc. of the 11th International IEEE Conference on Intelligent Transportation Systems, pages 1043 1048, 2008.

[25] K. Lee and H. Peng. Evaluation of automotive forward collision warning and collision avoidance algorithms. Vehicle System Dynamics, 43(10):735-751, 2005.

[26] C.-F. Lin, A. G. Ulsoy, and D. J. LeBlanc. Vehicle dynamics and external disturbance estimation for vehicle path prediction. IEEE Transactions on Control Systems Technology, 8:508-518, 2000.

[27] J. Lunze and B. Nixdorf. Representation of hybrid systems by means of stochastic automata. Mathematical and Computer Modeling of Dynamical Systems, 7:383-422, 2001.

[28] J. Maroto, E. Delso, J. Flez, and J. M. Cabanellas. Real-time traffic simulation with a microscopic model. IEEE Transactions on Intelligent Transportation Systems, 7(4):513-527, 2006.

[29] J. C. McCall and M. M. Trivedi. Video-based lane estimation and tracking for driver assistance: Survey, system, and evaluation. IEEE Transactions on Intelligent Transportation Systems, 7:20-37, 2006.
[30] B. T. Morris and M. M. Trivedi. Learning, modeling, and classification of vehicle track patterns from live video. IEEE Transactions on Intelligent Transportation Systems, 9:425-437, 2008.

[31] H. Ning, W. Xu, Y. Zhou, Y. Gong, and T. S. Huang. A general framework to detect unsafe system states from multisensor data stream. IEEE Transactions on Intelligent Transportation Systems, 11:4-15, 2010.

[32] F. Oniga and S. Nedevschi. Processing dense stereo data using elevation maps: Road surface, traffic isle, and obstacle detection. IEEE Transactions on Vehicular Technology, 59:1172-1182, 2010.

[33] A. Polychronopoulos, M. Tsogas, A. J. Amditis, and L. Andreone. Sensor fusion for predicting vehicles' path for collision avoidance systems. IEEE Transactions on Intelligent Transportation Systems, 8(3):549-562, 2007

[34] R. Rajamani. Vehicle Dynamics and Control. Springer, 2005.

[35] F. Rohrmüller, M. Althoff, D. Wollherr, and M. Buss. Probabilistic mapping of dynamic obstacles using Markov chains for replanning in dynamic environments. In Proc. of the IEEE/RSJ International Conference on Intelligent Robots and Systems, pages 2504-2510, 2008

[36] J. Schröder. Modelling, State Observation and Diagnosis of Quantised Systems. Springer, 2003.

[37] S. Sekizawa, S. Inagaki, T. Suzuki, S. Hayakawa, N. Tsuchida, T. Tsuda, and $\mathrm{H}$. Fujinami. Modeling and recognition of driving behavior based on stochastic switched ARX model. IEEE Transactions on Intelligent Transportation Systems, 8:593-606, 2007.

[38] T. Toledo. Integrated Driving Behavior Modeling. $\mathrm{PhD}$ thesis, Massachusetts Institute of Technology, 2003.

[39] D. Vasquez, T. Fraichard, and C. Laugier. Incremental learning of statistical motion patterns with growing hidden markov models. IEEE Transactions on Intelligent Transportation Systems, 10:403-416, 2009.

[40] A. L. Visintini, W. Glover, J. Lygeros, and J. Maciejowski. Monte Carlo optimization for conflict resolution in air traffic control. IEEE Transactions on Intelligent Transportation Systems, 7:470-482, 2006.

[41] S. Weinzierl. Introduction to Monte Carlo methods. Technical report, NIKHEF Theory Group Kruislaan 409, 1098 SJ Amsterdam, The Netherlands, 2000.

[42] Y. U. Yim and S.-Y. Oh. Modeling of vehicle dynamics from real vehicle measurements using a neural network with two-stage hybrid learning for accurate long-term prediction. IEEE Transactions on Vehicular Technology, 53:1076-1084, 2004.

[43] R. Yuster and U. Zwick. Fast sparse matrix multiplication. ACM Transactions on Algorithms, 1:2-13, 2005.

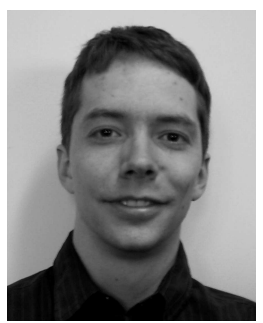

Matthias Althoff Matthias Althoff received the diploma engineering degree in Mechanical Engineering in 2005, and the Ph.D. degree in Electrical Engineering in 2010, both from the Technische Universität München, Germany. Currently he is a postdoctoral researcher in the department of Electrical and Computer Engineering at Carnegie Mellon University, Pittsburgh, USA. His research interests include (probabilistic) reachability analysis of continuous and hybrid systems, and the safety analysis of autonomous cars.

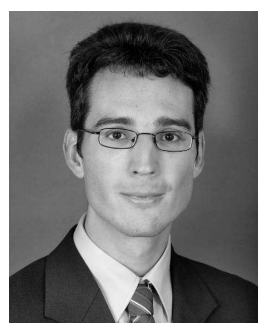

Alexander Mergel Alexander Mergel received the diploma engineering degree in Electrical Engineering in 2010 from the Technische Universität München, Germany. His research interests include Monte Carlo simulation, the safety assessment of autonomous cars, and optimal control of hybrid systems. 